\title{
Stratégies \\ vaccinales contre \\ le mélanome
}

> Le mélanome est une pathologie de plus en plus

François Ghiringhelli, Laurence Zitvogel

fréquente, sévère, pour laquelle les traitements antitumoraux standards sont peu efficaces. Étant donné la chimiorésistance et la radiorésistance intrinsèque de cette tumeur, d'une part, et d'autre part l'existence d'un rejet spontané, certes rare, de certains mélanomes, de grands espoirs se portent sur les techniques d'immunothérapie. Le développement récent de techniques de surveillance immunologique, l'«immunomonitorage», la caractérisation de nombreux antigènes tumoraux et la découverte de cytokines nécessaires à la culture ex vivo de cellules dendritiques ont ouvert de nouvelles voies thérapeutiques. Cet article fait le point sur les différentes stratégies de vaccination contre le mélanome. <

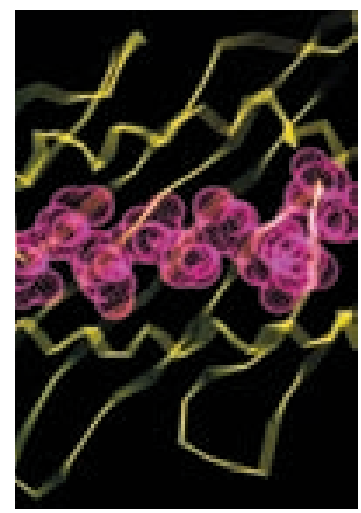

F. Ghiringhelli : Inserm U.517, Faculté de Médecine et Centre Régional de Lutte contre le Cancer Georges-Francois Leclerc, 7, boulevard Jeanne d'Arc, 21079 Dijon Cedex, France. L. Zitvogel : ERM 02-08 Inserm, Département de biologie clinique, Institut Gustave Roussy, 39, rue Camille Desmoulins, 94805 Villejuif Cedex, France.

I'IFN $\alpha$ en situation f.ghiringhelli@compaqnet.fr adjuvante [1]. En situation métastatique, I'IL-2 (interleukine-2) et I'IFN $\alpha$ entraînent dans $15 \%$ à $20 \%$ des cas une réponse clinique souvent partielle et de courte durée, même s'il existe de rares rémissions complètes prolongées.

L'incidence du mélanome est en augmentation tante, le traitement de choix restant la résection chirurgicale quand la maladie est localisée. Cependant, lorsque la lésion est profonde ou que des ganglions satellites sont envahis, les patients restent exposés au risque d'évolution métastatique; ils peuvent alors bénéficier d'un traitement par l'interféron $\alpha$ (IFN $\alpha$ ), qui diminue le risque de récidive [1]. La maladie métastatique nécessite quant à elle un traitement systémique : la chimiothérapie donne des taux de réponses d'environ $20 \%$, et n'augmente pas la médiane de survie, qui reste aux alentours de 9 mois [2].

Le système immunitaire joue un rôle dans l'évolution naturelle du mélanome : en effet, l'incidence du mélanome augmente en cas d'immunosuppression, certains mélanomes régressent spontanément et l'infiltration de la tumeur par les lymphocytes $T$ semble être un facteur de bon pronostic [3]. Toutes ces données ont amené à penser que des stratégies immunologiques vaccinales pourraient améliorer le pronostic des mélanomes localement évolués et métastatiques.

À l'heure actuelle, l'immunothérapie du mélanome se résume en pratique clinique à l'utilisation de

Article reçu le 8 juin 2005, accepté le 13 juillet 2005.

\section{Thérapies vaccinales passives par injection de lymphocytes T spécifiques}

La découverte de lymphocytes T infiltrant (TIL) les tumeurs et capables de détruire in vitro des cellules de mélanome a suggéré qu'un transfert passif de lymphocytes T activés ex vivo pourrait avoir un effet thérapeutique. Les premiers essais cliniques consistaient à injecter, en association avec des injections d'IL2, des lymphocytes $T$ provenant de la tumeur primaire et amplifiés par de fortes doses d'IL2. Ces procédés ont entraîné environ 20 \% de réponses objectives [4], mais l'expansion de lymphocytes T antitumoraux n'est possible que dans $40 \%$ des cas.

De nouvelles techniques permettent de mieux sélectionner les lymphocytes T antitumoraux: ainsi, les TIL peuvent être cultivés avec des cellules tumorales autologues ou allogéniques pour augmenter leur spécificité antitumorale; ils peuvent également être stimulés par des peptides issus d'antigènes tumoraux, pour induire une réponse clonale contre un antigène particulier; 
enfin, la transfection des cellules tumorales avec des molécules de costimulation, comme CD80, permet aussi d'améliorer la sélection et la prolifération des lymphocytes T [6]. Malgré toutes ces innovations, les thérapeutiques utilisant les TIL n'ont jamais montré de supériorité par rapport à I'IL-2 seule. Greenberg et ses collaborateurs ont apporté la preuve de principe de la migration préférentielle intratumorale des TIL réinjectés, et de l'intérêt de modifier génétiquement les lymphocytes T pour allonger leur survie, souvent courte in vivo [7]. Le groupe français de Jotereau et Dreno a également mené un essai clinique de phase II/III sur 88 patients atteints d'un mélanome de stade III en adjuvant, testant l'efficacité d'une thérapie par TIL expandus ex vivo + IL-2, contre un traitement par I'IL-2 seule : cette étude a montré le bénéfice thérapeutique, en survie sans récidive, de la ré-injection de TIL reconnaissant la tumeur autologue chez le sous-groupe de patients n'ayant qu'un seul ganglion envahi [8].

Enfin, l'intérêt de cette thérapeutique a été renouvelé par l'équipe de Rosenberg [9]: des patients ayant résisté à l'IL-2 ont été traités par transfert passif de lymphocytes sélectionnés pour leur capacité à reconnaître les cellules tumorales autologues après un prétraitement lympho-ablatif (cyclophosphamide + fludarabine). Ce traitement a entraîné 6 réponses objectives accompagnées d'effets auto-immuns sur 13 patients traités; une prolifération oligoclonale de clonotypes antitumoraux a été observée in vivo, migrant au sein de la tumeur. Bien que non dénuée de toxicité, la ré-injection de TIL accompagnés de CD4 ${ }^{+}$et de cytokines telles que l'IL2 (IL-15 ou IL-7) après conditionnement non myélo-ablatif semble efficace dans le mélanome de stade IV, et mérite d'être de nouveau testée de façon contrôlée.

\section{Immunothérapie active : la vaccination proprement dite}

Ces traitements d'immunothérapie active consistent à induire une réponse immunitaire $T$ primaire in vivo. Ces vaccins se répartissent en quatre catégories: cellules tumorales génétiquement modifiées (GMTV), protéines de choc thermique, peptides antitumoraux et cellules dendritiques chargées en antigènes tumoraux.

\section{Vaccination par cellules tumorales}

La vaccination par cellules tumorales consiste à injecter des cellules tumorales autologues ou allogéniques inactivées, associées à un adjuvant, dans le but de potentialiser la réponse immunitaire.

L'utilisation de cellules tumorales autologues est conceptuellement satisfaisante, car elle est à même d'en- traîner une réponse immunitaire polyclonale. Néanmoins, il faut noter que l'extraction et la purification de cellules tumorales présentent des difficultés techniques, et parfois seuls des macrophages ou des fibroblastes sont sélectionnés. Par ailleurs, à l'heure actuelle, cette technique n'a pas montré d'efficacité dans le mélanome: ainsi, Berd [10] n'obtient que $12 \%$ de réponses contre des mélanomes métastatiques. La découverte d'antigènes communs exprimés par les mélanomes et la difficulté que représente la purification de cellules tumorales ont conduit à utiliser en thérapeutique des lignées allogéniques de mélanome portant des antigènes fréquemment exprimés. Deux vaccins cellulaires établis à partir de lignées allogéniques semblent montrer une efficacité en situation adjuvante, et à un moindre niveau en situation métastatique, dans des études de phases II [11-13] ; ils sont actuellement en cours de validation dans le cadre d'une étude de phase III. La découverte que la transfection de cellules tumorales par un gène codant pour une cytokine (IL-2, IFN $\alpha$, GMCSF [granulocyte-macrophage colony-stimulating factor]), IL-12...) pouvait rendre ces cellules plus immunogéniques dans des modèles animaux a déclenché le lancement d'essais thérapeutiques: leurs résultats sont toutefois décevants pour l'instant [14], sauf en ce qui concerne l'utilisation de cellules transfectées avec le gène codant pour le GMCSF, qui s'accompagne de fortes réponses immunologiques locales et dans les tumeurs, et de quelques réponses cliniques [15].

\section{Vaccination par protéine de choc thermique}

Les protéines de choc thermique sont des protéines chaperons présentes dans le réticulum endoplasmique ou le cytosol, et jouant un rôle capital dans l'homéostasie cellulaire, notamment en protégeant les protéines intracellulaires de l'agrégation en cas de stress. Les concentrations de ces protéines peuvent augmenter en cas de stress comme I'hyperthermie ou le défaut en nutriment ou en oxygène.

Ces protéines semblent immunogéniques uniquement en système autologue : en effet, HSP70 et gp96 sont capables de porter des peptides antigéniques; chargées en peptides, elles peuvent être endocytées par les cellules dendritiques exprimant des récepteurs les reconnaissant et, de fait, induire leur maturation et le phénomène d'apprêtement de l'antigène, et sa présentation en association avec les molécules du complexe majeur d'histocompatibilité ( $\mathrm{CMH}$ ) de classe II, mais aussi de classe I [16]. Des essais thérapeutiques semblent confirmer I'intérêt de ces molécules: ainsi, une étude de phase I/II, utilisant des protéines gp96 isolées de la tumeur autologue, a montré $18 \%$ de réponses cliniques chez des patients porteurs de mélanomes métastatiques [17], résultats corroborés dans un modèle de tumeurs coliques par une autre équipe.

\section{Vaccination par peptide}

Depuis le clonage de MAGE-1 (melanoma-associated antigen 1), le premier antigène de tumeur identifié comme pouvant être reconnu par les lymphocytes $T$, de nombreux autres antigènes de tumeur ont été caractérisés. Des peptides provenant de ces antigènes peuvent être présentés par les molécules du CMH et induire une réponse immunitaire: les peptides caractérisés à l'heure actuelle proviennent essen- 
tiellement de protéines hyperexprimées ou mutées, ou de protéines de différenciation, et se lient essentiellement à la molécules HLA-*A0201, la plus représentée chez les sujets caucasiens.

Dans les essais cliniques, les peptides sont utilisés seuls ou, pour augmenter leur immunogénicité, en association avec des cytokines (IL-2, IL-12, GMCSF) ou des adjuvants (QS-21, adjuvant incomplet de Freund, ligands des récepteurs Toll) [18]. Ces vaccinations donnent une réponse immunologique chez certains patients, avec production de lymphocytes T CD8 spécifiques; ceux-ci sont détectables par la technique des tétramères, ou par des tests in vitro de type ELISPOT IFN $\gamma$, permettant de mettre en évidence des lymphocytes T spécifiques d'un antigène soit en recherchant leur capacité à se lier à un complexe $\mathrm{CMH} /$ peptide, soit en déterminant leur capacité à produire de l'IFN $\gamma$ après stimulation par le peptide [19]. D’après les résultats de plusieurs essais thérapeutiques, il semble que l'importance de la réponse immunologique soit corrélée aux réponses cliniques [20]. L'utilisation d'anticorps anti-CTLA-4 (cytotoxic T lymphocyte associated-4) montre une potentialisation de l'efficacité thérapeutique des vaccinations, mais au prix de réactions auto-immunes importantes (colites, uvéites, vitiligo) [21].

Le problème de la vaccination peptidique repose sur l'instabilité des peptides, sur la nécessité de réaliser le typage HLA du patient et celle de connaître le profil antigénique de la tumeur autologue. La vaccination par protéine de tumeur pallie ce problème et permet de développer une réponse polyclonale: des essais, effectués avec l'antigène tumoral NY-ESO-1 en association avec un adjuvant, montrent que ce protocole permet d'obtenir des réponses immunologiques et semble ralentir l'évolution tumorale [22].

\section{Vaccination par cellules dendritiques}

Les cellules dendritiques sont les seules cellules présentatrices d'antigènes capables d'initialiser des réponses lymphocytaires T primaires. Les cellules dendritiques murines chargées avec des antigènes tumoraux peuvent induire une protection antitumorale, voire une régression tumorale [23, 24]. Le premier type de cellules dendritiques étudié a été la cellule dendritique myéloïde, cellule de Langerhans (typiquement épidermique) ou cellule dendritique interstitielle (typiquement dermique). Suivant leur état de maturation, ces cellules sont capables d'induire une tolérance (stade «immature ») ou d'activer les lymphocytes (stade «mature ») via une production d'IL-12. L'autre type de cellules dendritiques est représenté par «les cellules dendritiques plasmacytoïdes », capables de produire rapidement de grandes quantités d'IFNa après contact viral. Ces cellules participent à l'immunité innée, en contrôlant les infections virales, et à l'immunité acquise, par l'induction d'une réponse T [25].

La découverte des cytokines (notamment le GMCSF, le TNF $\alpha$, I'IL-4 et I'IFN $\alpha$ ) a rendu possible la différenciation de ces cellules et/ou leur expansion ex vivo, à partir de monocytes ou de cellules souches hématopoiétiques circulantes, afin de tester leur intérêt en immunothérapie anticancéreuse [25]. Ces cellules peuvent être sensibilisées aux antigènes tumoraux par chargement direct, sur les molécules du $\mathrm{CMH}$ de classe I et II, de peptides synthétiques spécifiques, ou par chargement indirect de corps apoptotiques ou d'exosomes [26] tumoraux (cross priming). Les atouts de l'utilisation de ces peptides tumoraux sont la non-nécessité de prélèvement de cellules tumorales et la possibilité d'un suivi immunologique des lymphocytes T spécifiques par ELISPOT ou monitoring par tétramères. En revanche, le typage HLA du patient est obligatoire, et il existe un risque d'immunosélection potentielle de variants tumoraux CMH classe I négatifs ou de perte d'épitopes. L'utilisation de cellules tumorales comme source d'antigène présente quant à elle l'avantage d'induire des réponses immunitaires polyclonales contre des antigènes inconnus ou privés. En revanche, elle nécessite l'accès aux cellules tumorales et l'établissement d'une culture primaire de cellules tumorales autologues. Pour remédier à ce problème, certains ont utilisé des lignées allogéniques «certifiées », afin de standardiser le traitement d'un patient à l'autre.

Dans la plupart des études pilotes, qui incluent une centaine de patients, des réponses immunologiques avec apparition de lymphocytes $T$ spécifiques sont retrouvées, qui s'accompagnent toutefois de moins de $15 \%$ de réponses cliniques objectives. Seule l'équipe de Banchereau [27] a rapporté une stabilisation ou une régression chez 10 patients sur 18 porteurs de mélanomes métastatiques HLA-A2, traités avec des cellules dendritiques dérivées des cellules $C D 34^{+}$et chargées avec les antigènes MAGE-3, MART-1, tyrosinase et Gp100; ces réponses sont corrélées aux réponses biologiques mesurées par ELISPOT IFN $\gamma$.

Afin d'améliorer la présentation antigénique des cellules dendritiques, on peut induire une fusion entre les cellules dendritiques et les cellules tumorales [28], ou encore transfecter les cellules dendritiques avec I'ARN des cellules tumorales; toutefois, ces techniques ont donné encore peu de résultats.

Notre équipe a, quant à elle, utilisé des exosomes dérivés de cellules dendritiques : il s'agit de vésicules, sécrétées par les cellules dendritiques lors de leur différenciation, exprimant les molécules de CMH-I et - II, la protéine hsp 70 et des molécules de costimulation, ce qui les rend capables de présenter des antigènes tumoraux. Ces exosomes ont été chargés avec des peptides restreints par les molécules du CMH classe I et II tumoraux : la vaccination avec ces vésicules standardisables s'est révélée efficace in vitro, et encourageante in vivo [26-29].

\section{Conclusions et perspectives}

Malgré la multitude d'essais thérapeutiques de vaccination antimélanome, aucun protocole n'a obtenu de résultats assez convaincants pour modifier la thérapeu- 
tique actuelle du mélanome. La stratégie optimale de vaccination est résumée dans la Figure 1. Plusieurs points méritent toutefois d'être soulevés.

Si la phase d'initiation des réponses immunes est obtenue, il reste le problème de la phase effectrice de reconnaissance de la cellule tumorale et celui de la tolérance locale induite par la tumeur. La présence de lymphocytes $T$ suppresseurs ou $T$ régulateurs joue un rôle clef pour freiner l'efficacité des T effecteurs [30]: de nouvelles voies thérapeutiques visent donc à contrecarrer leur action, par l'utilisation d'anti-CTLA-4, de complexes anti-CD25/toxine diphtérique ou d'inhibiteurs du TGF- $\beta$.

Des études récentes ont montré, dans le mélanome, une fréquence très élevée des précurseurs de lymphocytes $T$ cytotoxiques spécifiques antitumoraux [31], dont l'expansion in situ peut être corrélée à la présence de cellules dendritiques matures au sein des tumeurs [32]. À l'inverse, des cellules dendritiques immunosuppressives ont été retrouvées dans certaines tumeurs, et seraient alors un facteur de mauvais pronostic. Au total, neutraliser les facteurs inhibiteurs locaux ou activer les cellules dendritiques locorégionales semble un objectif premier avant d'agir sur le priming des lymphocytes $T$ effecteurs dans la maladie métastatique. $\varepsilon$ n revanche, l'objectif du priming est probablement à maintenir dans le cadre de la maladie résiduelle (en situation adjuvante).
Par ailleurs, le faible nombre d'études contrôlées (phase II/III) réalisées et l'hétérogénéité des stades IV du mélanome rendent plus difficile la mise en évidence d'un bénéfice de la vaccination à ce stade. Enfin, l'analyse des réponses objectives n'est peut-être pas le meilleur paramètre alternatif de suivi d'efficacité. Puisque les techniques d'immunomonitorage restent délicates et indirectes aujourd'hui, l'objectif de «survie sans récidive » pourrait être plus adapté. $\diamond$

\section{SUMMARY}

\section{Melanoma immunotherapy}

Melanoma incidence increases and conventional antitumor therapies are often ineffective, encouraging the design of novel therapies. Several lines of evidence support the notion of an immunological control of melanoma growth. Based on this information, active immunotherapy (vaccination) and adoptive immunotherapy trials ( $T$ cell therapy) were conducted in metastatic melanoma patients. The proof of principle of effective immunotherapy was brought up by pionnering trials using tumor infiltrated lymphocytes in lymphodepleted recipients or anti-CTLA4 Ab leading to tumor eradication but also autoimmune diseases. With the identification and characterization of tumor antigens recognized by cytotoxic $T$ lymphocytes, the utilization of tumor rejection antigens along with adjuvants become available as tumor vaccines. The last five years have witnessed the emergence of dendritic cell based-vaccines that were efficient in priming and/or boosting T cell responses in normal volunteers and patients. This review highlights preclinical bases of cancer vaccines, their clinical development and discusses their limits. Correlations between immunomonitoring and tumor regressions await larger trials. $\diamond$

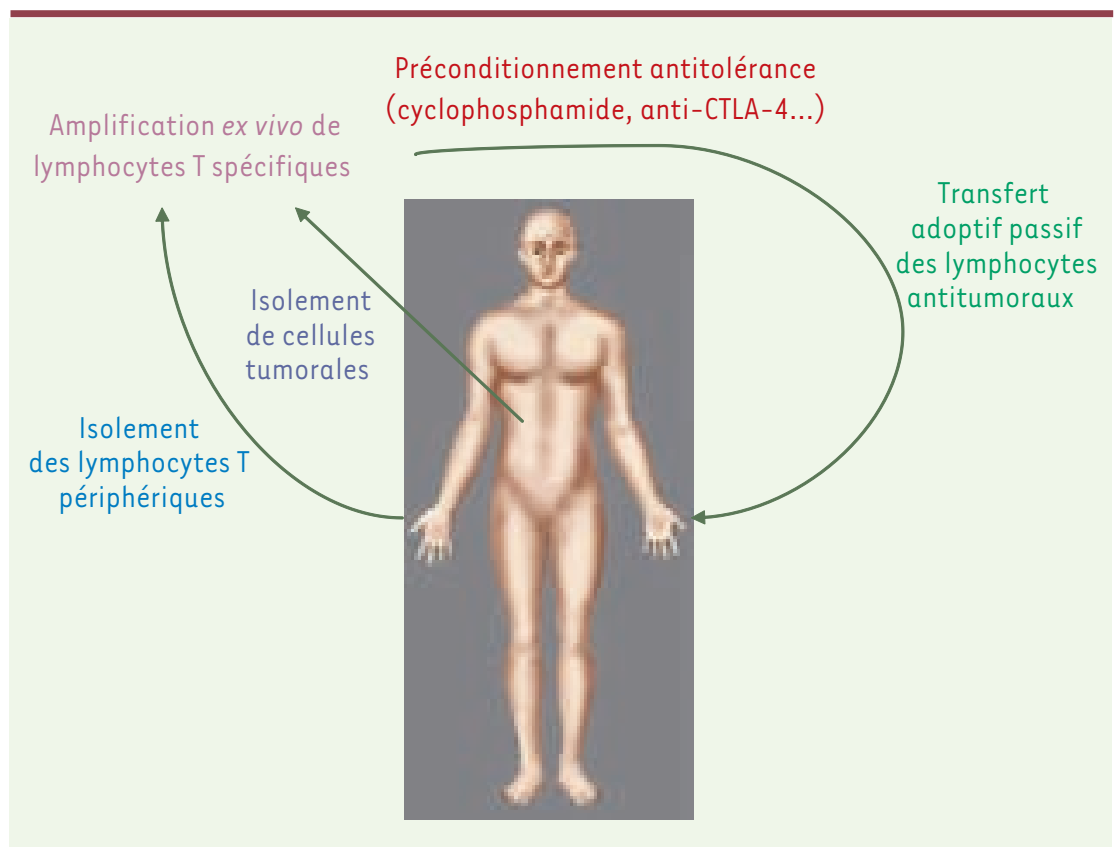

Figure 1. Stratégie optimale de vaccination antimélanome. CTLA-4: cytotoxic T lymphocyte associated -4

\section{RÉFÉRENCES}

1. Kirkwood JM, Strawderman MH, Ernstoff MS, et al. Interferon alpha-2b adjuvant therapy of highrisk resected cutaneous melanoma: the Eastern cooperative oncology group trial EST 1684. J Clin Oncol 1996; $14: 7-17$.

2. Tsao H, Atkins MB, Sober AJ. Management of cutaneous melanoma. N Engl J Med 2004 ; 351 : 998-1012.

3. Clark WH Jr, Elder DE, Guerry D $4^{\text {th }}$, et al. Model predicting survival in stage I melanoma based on tumor progression. J Natl Cancer Inst 1989; 81: 1893-904.

4. Rosenberg SA, Lotze MT, Muul LM, et al. Observations on the systemic administration of autologous lymphokine-activated killer cells and recombinant interleukin-2 to patients with metastatic cancer. N Engl J Med 1985 ; 313 : 1485-92.

5. Rosenberg SA, Dudley ME. Cancer regression in patients with metastatic melanoma after the transfer of autologous antitumor lymphocytes. Proc Natl Acad Sci USA 2004 ; 101 : 14639-45.

6. Dudley ME, Rosenberg SA. Adoptive-cell-transfer therapy for the treatment of patients with cancer. Nat Rev Cancer $2003 ; 3: 666-75$.

7. Yee C, Thompson JA, Byrd D, et al. Adoptive T cell therapy using antigen-specific $\operatorname{CD} 8^{+} \mathrm{T}$ cell clones for the treatment of patients with metastatic melanoma : in vivo persistence, migration, and antitumor effect of transferred T cells. Proc Natl Acad Sci USA 2002 ; 99 : 16168-73. 
8. Labarriere N, Pandolfino MC, Gervois N, et al. Therapeutic efficacy of melanoma-reactive TIL injected in stage III melanoma patients. Cancer Immunol Immunother 2002 ; 51 : 532-8.

9. Dudley ME, Wunderlich JR, Robbins PF, et al. Cancer regression and autoimmunity in patients after clonal repopulation with antitumor lymphocytes. Science $2002 ; 298: 850-4$

10. Berd D. M-Vax : an autologous, hapten-modified vaccine for human cancer. Exp Opin Ther $2002 ; 2: 335-42$.

11. Mitchell MS, Rechtman DJ, Von Eschen KB. A randomized phase III trial of Melacine versus combination chemotherapy in patients with disseminated melanoma. Can J Infect Dis 1995 ; $6($ suppl C) : 347 .

12. Hsueh $\varepsilon$, Gupta R, Qi K, Morton D. Correlation of specific immune responses with survival in melanoma patients with distant metastases receiving polyvalent melanoma cell vaccine. J Clin Oncol $1998 ; 16: 2913-20$.

13. Hsueh EC, Essner R, Foshag LJ, et al. Prolonged survival after complete resection of disseminated melanoma and active immunotherapy with a therapeutic cancer vaccine. J Clin Oncol 2002; $20: 4549-54$.

14. Parmiani G, Rodolfo M, Melani C. Immunological gene therapy with ex vivo gene-modified tumor cells: a critique and a reappraisal. Hum Gene Ther $2000 ; 11: 1269-75$.

15. Soiffer R, Hodi FS, Haluska F, et al. Vaccination with irradiated, autologous melanoma cells engineered to secrete granulocyte-macrophage colony-stimulating factor by adenoviralmediated gene transfer augments antitumor immunity in patients with metastatic melanoma. J Clin Oncol $2003 ; 21: 3343-50$.

16. C Castelli, L Rivoltini, F Rini. Heat shock proteins : biological functions and clinical application as personalized vaccines for human cancer. Cancer Immunol Immunother 2004 : $53: 227-233$

17. Belli F, Testori A, Rivoltini L, et al. Vaccination of metastatic melanoma patients with autologous tumor-derived heat shock protein gp96-peptide complexes : clinical and immunologic findings. J Clin Oncol $2002 ; 20: 4169-80$.

18. Parmiani G, Castelli C, Dalerba P, et al. Cancer immunotherapy with peptide-based vaccines what have we achieved? Where are we going ? J Natl Cancer Inst 2002 ; $94: 805-18$.

19. Daniel $\varepsilon$, Speiser A, Mikaël J, et al. Evaluation of melanoma vaccines with molecularly defined antigens by ex vivo monitoring of tumor-specific T cells. Semin Cancer Biol 2003 ; 13 : 461-72.

20. Lonchay $C$, van der Bruggen P, Connerotte $T$, et al. Correlation between tumor regression and $T$ cell responses in melanoma patients vaccinated with a MAGE antigen. Proc Natl Acad Sci USA $2004 ; 101$ (suppl : 14631-8).

21. Sanderson K, Scotland R, Lee P et al. Autoimmunity in a phase I trial of a fully human anticytotoxic T-lymphocyte antigen-4 monoclonal antibody with multiple melanoma peptides and Montanide ISA 51 for patients with resected stages III and IV melanoma. J Clin Oncol $2005 ; 23: 741-50$.

22. Davis ID, Chen W, Jackson $\mathrm{H}$, et al. Recombinant NY-ESO-l protein with Iscomatrix adjuvant induces broad integrated antibody and CD4 and CD8 T cell responses in humans. Proc Natl Acad Sci USA $2004 ; 101$ : 10697-702.
23. Thurner B, Haendler I, Roder C, et al. Vaccination with MAGE-3AI peptide-pulsed mature monocyte-derived dendritic cells expands specific cytotoxic T cells and induces regression of some metastases in advanced stages IV melanoma. J Exp Med 1999; 190 : 1669-78.

24. Chang AE, Redman BG, Whitfield JR, et al. A phase I trial of tumor lysatepulsed dendritic cells in the treatment of advanced cancer. Clin Cancer Res $2002 ; 8$ : 1021-32.

25. Shortman K, Liu YJ. Mouse and human dendritic cell subtypes. Nat Rev Immunol 2002 ; 2 : 151-61.

26. Wolfers J, Lozier A, Raposo G, et al. Tumor-derived exosomes are a source of shared tumor rejection antigens for CTL cross-priming. Nat Med 2001 ; $7: 297-303$.

27. Banchereau J, Palucka AK, Dhodapkar M, et al. Immune and clinical responses in patients with metastatic melanoma to $\mathrm{CD} 34^{+}$progenitorderived dendritic cell vaccine. Cancer Res 2001; 61:6451-8

28. Paczesny S, Ueno H, Fay J, et al. Dendritic cells as vectors for immunotherapy of cancer. Semin Cancer Biol $2003 ; 13: 439-47$.

29. Escudier B, Dorval T, Chaput N, et al. Vaccination of metastatic melanoma patients with autologous dendritic cell (DC) derivedexosomes: results of the first phase I clinical trial. J Transl Med2005; $3: 10$

30. Ghiringhelli F, Larmonier N, Schmitt $\varepsilon$, et al. CD4+CD25+ regulatory $T$ cells suppress tumor immunity but are sensitive to cyclophosphamide which allows immunotherapy of established tumors to be curative. Eur J Immunol 2004 ; 34 : 336-44.

31. Germeau C, Ma W, Schiavetti F, et al. High frequency of antitumor T cells in the blood of melanoma patients before and after vaccination with tumor antigens. J Exp Med $2005 ; 201: 241-8$

32. Movassagh M, Spatz A, Davoust J, et al. Selective accumulation of mature DC-Lamp ${ }^{+}$dendritic cells in tumor sites is associated with efficient T-cell-mediated antitumor response and control of metastatic dissemination in melanoma. Cancer Res $2004 ; 64: 2192-8$
TIRÉS À PART

L. Zitvogel

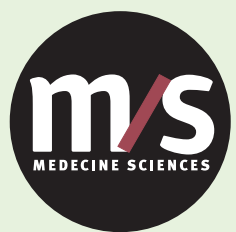

Tarifs d'abonnement M/S - 2006

Abonnez-vous

à Médecine/Sciences
> Depuis 20 ans, grâce à $m / s$, vous vivez en direct les progrès des sciences biologiques et médicales

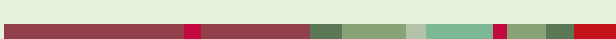

Bulletin d'abonnement page 130 dans ce numéro de $\mathrm{m} / \mathrm{s}$
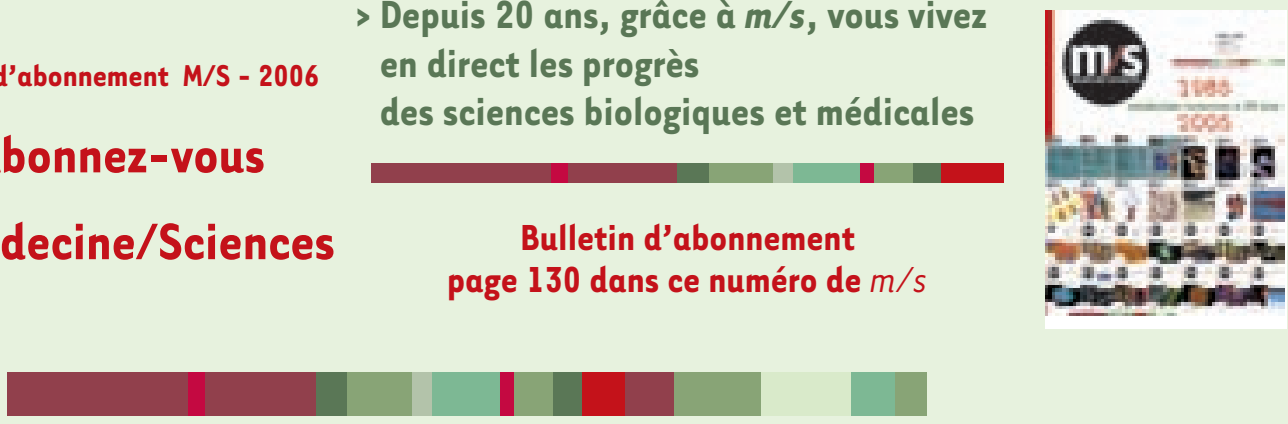